\title{
Physical activity and health related quality of life
}

Nana Kwame Anokye ${ }^{1 *}$, Paul Trueman ${ }^{1}$, Colin Green ${ }^{2}$, Toby G Pavey ${ }^{3}$ and Rod S Taylor ${ }^{2}$

\begin{abstract}
Background: Research on the relationship between Health Related Quality of Life (HRQoL) and physical activity (PA), to date, have rarely investigated how this relationship differ across objective and subjective measures of PA. The aim of this paper is to explore the relationship between HRQoL and PA, and examine how this relationship differs across objective and subjective measures of PA, within the context of a large representative national survey from England.

Methods: Using a sample of 5,537 adults (40-60 years) from a representative national survey in England (Health Survey for England 2008), Tobit regressions with upper censoring was employed to model the association between HRQOL and objective, and subjective measures of PA controlling for potential confounders. We tested the robustness of this relationship across specific types of PA. HRQoL was assessed using the summary measure of health state utility value derived from the EuroQol-5 Dimensions (EQ-5D) whilst PA was assessed via subjective measure (questionnaire) and objective measure (accelerometer- actigraph model GT1M). The actigraph was worn (at the waist) for 7 days (during waking hours) by a randomly selected sub-sample of the HSE 2008 respondents (4,507 adults - 16 plus years), with a valid day constituting 10 hours. Analysis was conducted in 2010.
\end{abstract}

Results: Findings suggest that higher levels of PA are associated with better HRQoL (regression coefficient: 0.026 to 0.072). This relationship is consistent across different measures and types of PA although differences in the magnitude of HRQoL benefit associated with objective and subjective (regression coefficient: 0.047) measures of PA are noticeable, with the former measure being associated with a relatively better HRQOL (regression coefficient: 0.072).

Conclusion: Higher levels of PA are associated with better HRQoL. Using an objective measure of PA compared with subjective shows a relatively better HRQoL.

Keywords: Health related Quality of Life, Objective measure, Self reports, Physical activity, EQ-5D

\section{Background}

Physical activity (PA) is associated with reduced risk for health conditions including coronary heart disease, cancers, diabetes, and stroke [1-3]. Despite interventions to increase PA in the English general population, the Health Survey for England (HSE) (2008) showed that only 39\% of men and 29\% of women in England are meeting the recommended level to be considered 'physically active' as defined by guidance from the Chief Medical Officer; only $6 \%$ of men and $4 \%$ of women, however, met the recommended level when PA was objectively measured [4].

\footnotetext{
* Correspondence: Nana.Anokye@brunel.ac.uk

${ }^{1}$ Health Economics Research Group (HERG), Brunel University, Uxbridge, Middlesex, UB8 3PH, England

Full list of author information is available at the end of the article
}

Health Related Quality of Life (HRQoL), including the physical and mental well-being of an individual, is an important concept in health research and can help to inform decisions on prevention and treatment of ill health [5]. Estimates of the relationship between HRQoL and particular health states have served as inputs for economic evaluations intended to inform resource allocation decisions [6]. From a public health perspective a better understanding of how healthy lifestyles, such as uptake of PA, can influence HRQoL might help to inform policy intended to incentivise PA in the general population [5]. Evidence on the association between HRQoL and PA in the general population is limited as research, to date, has focused on specific interventions or populations with chronic conditions [7]. The dearth of evidence perhaps partly explains why economic evaluation of exercise interventions, to date, has rarely 
accounted for HRQoL gains directly attributable to increased exercise [8], so called process utility, and rather has focussed on the long-term effects of sustained PA on the incidence of chronic conditions.

A recent systematic review [7] showed that the few studies that have attempted to address this gap in knowledge, found a positive association between HRQoL and PA. The limited evidence base is, however, considered weak as the studies have methodological issues with respect to the measurement of PA $[7,9]$. The studies mainly used subjective (self-reported) measures of PA without adequate validation. Some commentators argue that regardless of appropriate validity and reliability tests, the use of subjective measures of PA are subject to overestimation [10]. The few studies [11,12] that used objective measures, however, relied on cardio-respiratory fitness, which unlike electronic devices (e.g. accelerometers) is not a direct measure of PA. As being a biological phenotype, cardio-respiratory fitness is influenced by both PA level and genetics, and the consequent interaction of both [7]. A notable exception is Hamer and Stamatakis [9] that used accelerometer data to explore the association between objectively assessed PA and subjective measures of well-being.

The present study is unique because it uses both objective and subjective measures of PA, and focuses on a broader measure of health status as it is the common endpoint in economic evaluation of public health interventions. The paper aims to explore the relationship between HRQoL and PA, and examines how this relationship differs across objective and subjective measures of PA, within the context of a large representative national survey from England. This study focuses on adults aged 40-60 years in order to: (a) relate the findings more directly to current evidence on exercise referral interventions in the UK as the literature centres on this age group; and (b) reflect the broader objectives of the programme of research exploring those interventions [8]. This research is part of a larger programme of research that explored the effectiveness and cost effectiveness of exercise referral schemes in the United Kingdom [8].

\section{Methods}

\section{Data}

Data came from the HSE, a routine cross sectional survey that draws a nationally representative sample of persons residing in private households in England, which is openly available. The sample and focus of the survey vary each year. Data from the 2008 survey was used in this study and included a sample of 9,191 households with 15,102 adults aged 16 or over, and a total child sample of 7,521. Households were sampled proportionately across the 9 Government Office regions of
England. Sampling was based on a multi-stage stratified random sampling design that used the postcode address file as a sampling frame. To improve power of analysis, boost samples and sampling weights were employed appropriately [4]. The primary focus of HSE 2008 was PA and fitness. The method of data collection involved faceto-face interviews, self-completion, clinical and physical measurements (including objective measurements of PA via accelerometers). Tools for data collection were validated in a pilot study conducted in 2007 prior to the main survey (further details can be found in Craig et al. [4]). To compensate for seasonal variation in responses, the time period for interviews covered JanuaryDecember 2008. This study draws on 5,537 observations that constitute 40-60 year olds among the adult sample.

\section{HRQoL}

HRQoL is measured in the HSE using the summary measure of health state utility value derived from the validated EuroQol-5 Dimensions (EQ-5D) [13]. These utility scores were generated using the descriptive system of the EQ-5D questionnaire (UK version), a standard HRQoL instrument with preference weights which are attached to combinations of responses. The EQ-5D descriptive system describes HRQoL in five dimensions (i.e. mobility, self-care, usual activities, pain/discomfort, and anxiety/depression) with each dimension including three levels: no problems, some/moderate problems, and severe/extreme problems. Different health states are created from the responses to the descriptive system of the EQ-5D by combining one level from each of the dimensions. A tariff is then applied to these health states to generate utility scores [14]. The utility scores usually range from ' 1 ' (perfect health) to ' 0 ' (death), with states perceived to be worse than death having a negative utility score.

\section{Physical activity}

PA was accessed via a composite indicator, reflecting a combination of types of PA (i.e. walking, housework, occupational activity, and sports and exercise) and captured through subjective, and objective measurements. In HSE 2008, the subjective measure of PA was assessed through a validated questionnaire and the objective measure the most widely used accelerometer, actigraph (model GT1M), which is relatively portable (due to its small size) and associated with low running costs [4]. Accelerometer is a favoured method of objective measurement of physical activity given that it has an increased capacity to capture varied movements [4]. A pilot study was conducted to examine the feasibility of using the actigraph was and it was found to perform well. In the main survey, the actigraph was worn (at the waist) by a randomly selected sub-sample of the HSE 
2008 respondents (4,507 adults -16 plus years). Respondents were told to wear the actigraph during waking hours for 7 consecutive days following an initial discussion with interviewers who explained the use of the device and gave respondents phone numbers to call for assistance when faced with difficulties using the device. A book was given to participants to log daily use of the actigraph (duration of use/non-use). Daily use was considered 'valid' if the actigraph was worn for at least 10 hours. Kine software (3.0.98) was used to analyse the raw accelerometry data to generate a standardised measures. Further details on the use of accelerometer in the HSE 2008 can be found in Craig et al. [4].

To test the robustness of the relationship between PA and HRQoL across specific types of PA, separate measures were included for specific types of PA (i.e. walking, and sports and exercise). Data for walking included selfreports on all walking (regardless of intensity) ranging from country walks, walking to and from work/school, and any other walks. Sports and exercise covered selfreports on activities such as swimming, cycling, aerobics, workout at a gym, running, team sports, and press/situps. These two types of PA were chosen and analysed separately because they reflect the main types of exercise referral interventions [8].

Each PA measure was operationalised as a binary variable that takes the value of one if 'physically active' (minimum of 90 minutes of at least moderate intensive PA was undertaken per week) or zero otherwise (not physically active). This definition of being 'physically active' is consistent with the literature on exercise referral interventions and PA for health guidance [15]. Based on previous research $[11,12,16]$, we hypothesise that being 'physically active' would be positively correlated with HRQoL.

\section{Covariates}

The covariates were socio-demographic, economic, health and other variables that in previous research had been shown to correlate with HRQoL: gender, age, income, educational qualification, employment status, ethnicity, marital status, house tenure, smoking status, drinking status, region of residence, urbanisation, general health status, BMI, morbidities (e.g. problems with heart, muscoskeletal, ear, vision, mental, hypertension, stroke, diabetes), psycho-social wellbeing (measured via General Health Questionnaire scores) [17-23].

\section{Analysis}

Means (standard deviation - SD) and proportions were calculated for continuous and categorical data respectively. Chi square and Fischer's exact tests were used to check the association between HRQoL (dependent variable) and dummy variables representing item non- response for independent variables in order to examine the mechanisms under which the missing data occurred (i.e. missing completely at random or not) [24]. If the pattern of missing data did not occur completely at random (i.e. HRQoL is significantly associated with item non-response for independent variable(s), a regressionbased imputation method was used to replace missing values of continuous variables and a dummy variable specifying item-non response added. For the categorical variables, item non-response was included in the omitted category and a dummy variable for item nonresponse created [25].

To account for censoring in the measurement of HRQoL [26], Tobit regressions with upper censoring at 1.0 and robust standard errors were employed to model the relationship between HRQoL and PA controlling for covariates. Separate models were fitted for each indicator of PA to avoid unstable estimates resulting from the collinearity among those indicators. Hence four different models were estimated: model 1 (walking); model 2 (sport and exercise); model 3 (objective measurement); model 4 (subjective measurement). Hereafter, the models will be referred to by these names. Each model had two versions: (a) model excluding missing observations; and (b) model including imputed missing observations. To allow comparability between subjective and objective measures of PA, models 3 and 4 were estimated using the same sample (i.e. those with data for both subjective and objective measures).

The models were estimated with sampling weights that were calculated as the inverse of the probability of being a respondent in a household multiplied by the household weight which accounts for non-responding households [4]. Specification errors and goodness of fit of models were examined using the linktest [27], and Akaike Information Criteria (AIC) and Bayesian Information Criteria (BIC) [28] respectively. Pseudo $\mathrm{R}^{2}$ was computed by calculating the $R^{2}$ between the predicted and observed values [29] and multicollinearity among independent variables was assessed [30,31]. Threshold for statistical significance was set at $\leq 0.10$ and analysis was undertaken in 2010 using Stata version 10.

\section{Results}

\section{Description of sample}

Table 1 shows that the mean health state utility score (HQRoL) was 0.86 (Standard Deviation: 0.23; upper limit:1; lower limit:-0.484) and few individuals had limiting illness (23\%). The proportion of 'physically active' individuals ranged from $12 \%$ (via objective measurement) to $45 \%$ (via subjective measurement). Based on self-reports, $46 \%$ of respondents whose physical activity were objectively measured $(n=873)$ were physically active compared with $44 \%$ for those who did not provide 
Table 1 Descriptive statistics of variables (adjusted for missing observations)

\begin{tabular}{|c|c|c|}
\hline Variables & Observations & Mean(SD) $/ \%$ \\
\hline \multicolumn{3}{|l|}{ Dependent variable (HRQoL) } \\
\hline EQ-5D & 5453 & $0.86(0.23)$ \\
\hline Missing & 84 & 1.5 \\
\hline \multicolumn{3}{|l|}{ Independent variables (PA) } \\
\hline \multicolumn{3}{|l|}{ Walking } \\
\hline Active & 873 & 15.8 \\
\hline Inactive & 4664 & 84.2 \\
\hline \multicolumn{3}{|l|}{ Sports and exercise } \\
\hline Active & 660 & 11.9 \\
\hline Inactive & 4877 & 88.1 \\
\hline \multicolumn{3}{|l|}{ Objective measurement* } \\
\hline Active & 102 & 11.5 \\
\hline Inactive & 783 & 88.5 \\
\hline Missing & 4652 & 84 \\
\hline \multicolumn{3}{|l|}{ Subjective measurement ${ }^{* *}$} \\
\hline Active & 2452 & 44.4 \\
\hline Inactive & 3067 & 55.6 \\
\hline Missing & 18 & 0.3 \\
\hline \multicolumn{3}{|l|}{$\begin{array}{l}\text { Independent variables } \\
\text { (covariates) }\end{array}$} \\
\hline Age & 5537 & $50(6.2)$ \\
\hline \multicolumn{3}{|l|}{ Gender } \\
\hline Male & 2519 & 45.5 \\
\hline Female & 3018 & 54.5 \\
\hline \multicolumn{3}{|l|}{ Marital status } \\
\hline Other & 30 & 0.5 \\
\hline Married (living with partner) & 3618 & 65.3 \\
\hline Single & 735 & 13.3 \\
\hline Separated & 208 & 3.8 \\
\hline Divorced & 816 & 14.7 \\
\hline Widowed & 135 & 2.4 \\
\hline Income & 4535 & $35591.2(29210)$ \\
\hline Missing & 1002 & 18.1 \\
\hline $\begin{array}{l}\text { Income (missing observations } \\
\text { imputed for) }\end{array}$ & 5537 & $35008.3(26987.7)$ \\
\hline \multicolumn{3}{|l|}{ Drink alcohol } \\
\hline Yes & 4702 & 84.9 \\
\hline No & 823 & 14.9 \\
\hline Missing & 12 & 0.2 \\
\hline \multicolumn{3}{|l|}{ Smokers } \\
\hline Yes & 1206 & 21.8 \\
\hline No & 1926 & 34.8 \\
\hline Missing & 2405 & 43.4 \\
\hline BMI & & \\
\hline
\end{tabular}

Table 1 Descriptive statistics of variables (adjusted for missing observations) (Continued)

\begin{tabular}{|c|c|c|}
\hline Underweight (under 18.5) & 30 & 0.5 \\
\hline Normal (18.5 - 25) & 1487 & 26.9 \\
\hline Overweight $(25-30)$ & 1885 & 34 \\
\hline Obese (30+) & 1418 & 25.6 \\
\hline Missing & 717 & 13 \\
\hline \multicolumn{3}{|l|}{ Ethnicity } \\
\hline White & 5029 & 90.8 \\
\hline Mixed & 44 & 0.8 \\
\hline Asian & 260 & 4.7 \\
\hline Black & 140 & 2.5 \\
\hline Chinese & 28 & 0.5 \\
\hline Other & 17 & 0.3 \\
\hline Missing & 19 & 0.3 \\
\hline \multicolumn{3}{|l|}{ Education } \\
\hline Nvq4/nvq5/degree or equivalent & 1228 & 22.2 \\
\hline Higher education below degree & 746 & 13.5 \\
\hline Nva3/GCE 'A' level equivalent & 749 & 13.5 \\
\hline Nvq2/GCE 'O' level equivalent & 1404 & 25.4 \\
\hline Nva1/CSE other grade equivalent & 239 & 4.5 \\
\hline Foreign/other & 53 & 1.0 \\
\hline No qualification & 1102 & 19.9 \\
\hline Missing & 16 & 0.3 \\
\hline \multicolumn{3}{|l|}{ Employment status } \\
\hline Employed & 4215 & 76.1 \\
\hline Unemployed & 163 & 2.9 \\
\hline Retired & 259 & 4.7 \\
\hline Other economically inactive & 884 & 16 \\
\hline Missing & 16 & 0.3 \\
\hline
\end{tabular}

*The distribution of objective measurement (missing observations imputed for) is: active $(111 ; 2 \%)$ inactive $(5426 ; 98 \%){ }^{* *}$ The distribution of subjective measurement (missing observations imputed for) is: active (2465; 44.5\%) inactive $(3072 ; 55.5 \%)$. If restricted to observations with values for objective measurement, the distribution of subjective measurement (missing observations imputed for) is: active $(408 ; 46.1 \%)$ inactive $(477 ; 53.9 \%)$.

such data $(\mathrm{n}=4652)$. Majority of the sample were White (91\%), female (55\%), married and living with partners (66\%), educated (81\%), and employed (76\%). Few people were obese $(26 \%)$ or smokers $(22 \%)$ though majority (85\%) were 'drinkers of alcohol'. Average annual household income was $£ 35,591$ (SD: 29,210).

\section{Missing observations}

HQRoL had 84 missing observations (2\%), and these were dropped creating an effective sample of 5,453 observations. All the independent variables except walking; sports and exercise; age; marital status; region of residence and urbanisation had missing observations. Most variables had around $1 \%$ of missing data. The 
objective measure of PA had more missing observations (84\%) because as per HSE procedures only a random subset of the sample was selected for the objective measurement although the subjective measurement was based on the entire adult sample.

The HQRoL for individuals, who had missing values for social class; BMI; and smoking status, were significantly different from those who did not. However, HQRoL for individuals with missing values for the indicators of PA were not different from those who did not. Hence findings on the association between PA and HRQoL is not expected to be significantly affected by the inclusion (or not) of missing observations.

\section{Regression models}

Table 2 shows the reduced regression models estimating the correlation between PA and HRQoL, controlling for covariates. Results are presented for models (excluding missing observations) because they provide better fit and specification, and were consistent with those of models that included missing observations.

Higher levels of PA are associated with better HRQoL. Compared with inactive individuals, being 'physically active' via objective or subjective measure was associated with a higher HRQoL but the objective measure was associated with $53 \%$ extra HRQoL gain (coefficient $(r): 0.072)$ relative to the latter (coefficient $(r): 0.047)$.
The positive association between HQRoL and PA was consistent across specific types of PA as individuals who were 'physically active' via sports and exercise (coeffi$\operatorname{cient}(r): 0.034)$ or walking (coefficient $(r): 0.026)$ had better HRQoL than inactive individuals.

\section{Model diagnostics}

The specification error tests show that the models had good specification and that additional statistically significant regressors could only be found by chance (Table 3 ). The models' estimates could be considered stable as no sign of multicollinearity was found, with average variance inflation factors and tolerance levels at 1.2 and 0.8 respectively. A reasonable proportion (between $10 \%$ and $40 \%$ ) of variation in HRQoL was explained by the models as indicated by the pseudo $\mathrm{R}^{2}$. Model 3 appears to have the best fit according to Akaike Information Criteria (AIC) and Bayesian Information Criteria (BIC) values.

\section{Discussion}

The results support the hypothesis that higher levels of PA are associated with better HRQoL. This relationship is consistent across different measures and types of PA. Participation in walking and sports and exercise are correlated with a modest effect on HRQoL. However, differences in the magnitude of HRQoL benefit associated with objective and subjective measures of PA are

Table 2 Estimation results of regression models (reduced models without missing observations)

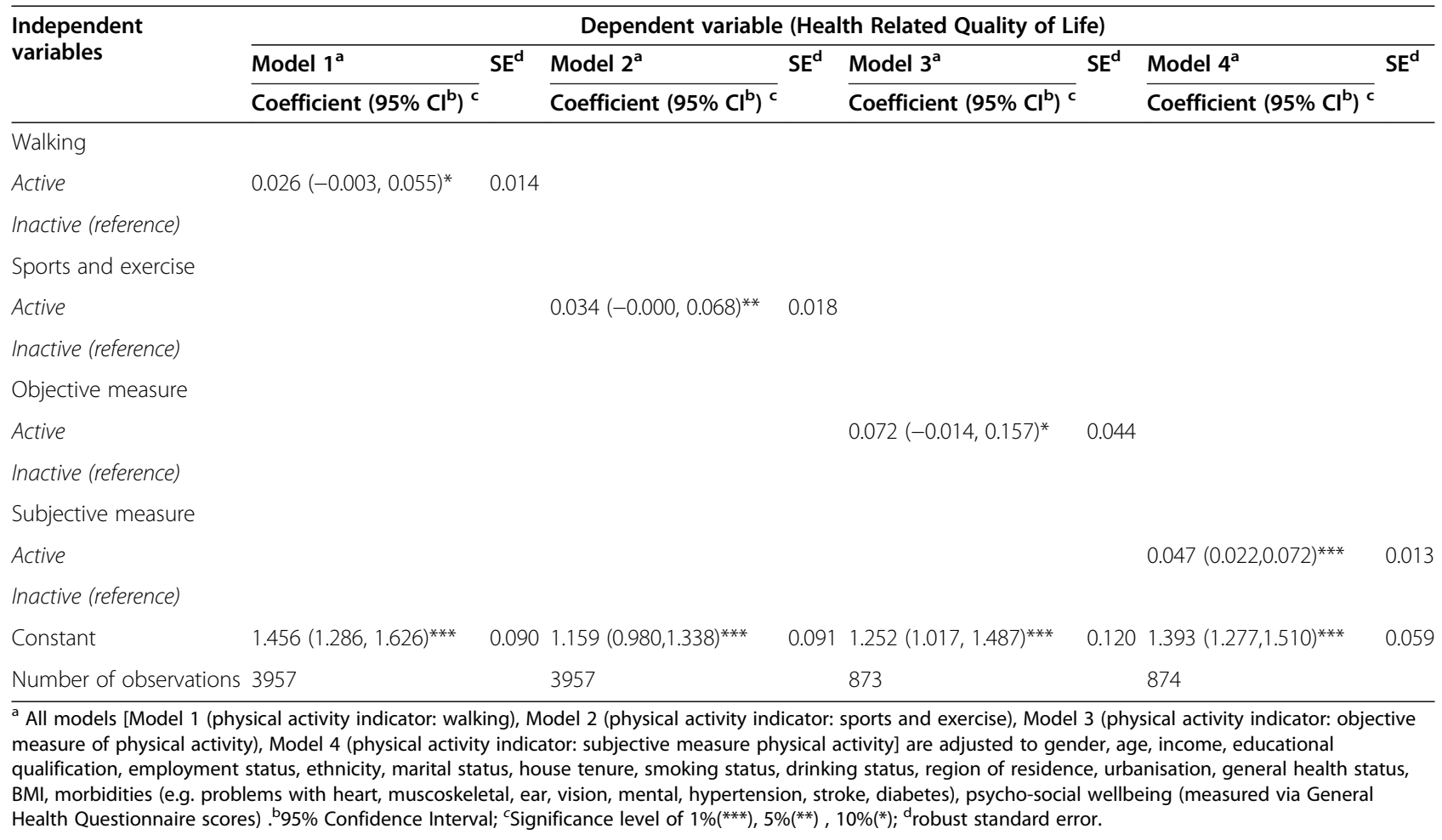


Table 3 Model diagnostics

\begin{tabular}{|c|c|c|c|c|c|c|}
\hline \multirow[t]{2}{*}{ Models } & \multicolumn{2}{|c|}{ Multicollinearity tests } & \multirow{2}{*}{$\frac{\text { Specification test }}{P>|t|}$} & \multirow[t]{2}{*}{ Pseudo $\mathrm{R}^{2}$} & \multirow[t]{2}{*}{ AIC } & \multirow[t]{2}{*}{ BIC } \\
\hline & VIF & Tolerance & & & & \\
\hline Model 1 & $1.05-1.45$ & $0.68-0.97$ & 0.063 & 0.429 & 3247.5 & 3360.6 \\
\hline Model 2 & $1.04-1.55$ & $0.65-0.95$ & 0.084 & 0.430 & 2697.7 & 2798.3 \\
\hline Model 3 & $1.00-1.22$ & $0.82-0.99$ & 0.845 & 0.092 & 841.7 & 889.5 \\
\hline Model 4 & $1.06-1.23$ & $0.81-0.94$ & 0.205 & 0.446 & 1936.9 & 2050.1 \\
\hline
\end{tabular}

noticeable, with the former measure being associated with a relatively better HRQoL. An explanation for this could be that people tend to over-report their participation levels in PA [32], for example, 90 minutes of at least moderate intensive PA captured via a subjective measure might actually equate to a shorter duration or a less intensive period of exercise when measured objectively. The potential impact of this is that it yields a downward bias in econometric estimation with respect to subjective measures of $\mathrm{PA}$, meaning that the link between HRQoL and PA is underestimated. This is further supported by descriptive statistics that indicate that people considered physically active via objective measure had slightly better mean HRQoL value (0.918) than individuals considered physically active via selfreports (0.916).

A key question is whether in order to achieve more accurate estimations, objective rather than subjective measures ought to be favoured. We are inclined towards a cautious approach, allowing for further corroboration of the findings because of a number of reasons. First, there was discrepancy in the reference periods of data collection for the two measures, subjective data was based on four weeks prior to survey date while objective data was based on a seven day period after the survey date. Second, the objective measure used herein, accelerometry, do not capture all types of PA with equivalent precision tending to underestimate activities like cycling and walking up stairs [4].

It is important that the limitations of the analysis are acknowledged. First, as a cross sectional study, the findings point to an association between HRQoL and PA and that no conclusions on causality can be drawn. Therefore, we cannot conclude whether participation in PA leads to higher HRQoL or improved HRQoL leads to a lifestyle (e.g. absence of limiting illnesses) more enabling for participation in PA. Nonetheless, the consistency of our findings across different types of PA strengthens the need for further research into the causal pathways between PA and HRQoL using longitudinal datasets. Second, the findings may not be generalisable to other age groups given the emphasis on adults aged 4060 years. However, the findings are consistent with previous evidence on the relationship between PA and HRQoL $[11,12,16]$. Further confidence can be drawn from the findings because all regression models had good specification and fit.

The findings suggest that there is a relationship between PA and HRQoL. However, the nature of the study means that it is not possible to accurately dissect the nature of this relationship. It is important to consider the constituents of the HRQoL gains associated with PA captured in this study. Participation in PA generally tends to be associated with two main types of benefits i.e. physical benefits (reduced risk for ill-health conditions, improved fitness levels) and psychological benefits (e.g. mental simulation during participation, improved psychological health) [33,34]. Given the cross sectional nature of our study and the fact that ill-health conditions were controlled for in the analysis, the benefits captured may more likely be psychological benefits. However, we are not able to determine the exact nature of these benefits. For example, the improvements in HRQoL associated with PA might be considered to be 'process benefits' that arise from engagement in PA. These might occur due to the process of participating (increased social interactions resulting from group participation or time spent outdoors), improved self-esteem (e.g. positive perceptions of competences and the physical-self) or biologic mechanisms (increased endorphin levels as a result of PA). Further research is needed to understand the relationship between PA and HRQoL and this may need to comprise both quantitative and qualitative methods.

Related to this, is the degree to which these benefits are lasting which also warrants further exploration as its crucial to how HRQoL gains are converted into outcomes (e.g. quality adjusted life years or 'QALY's') in an economic evaluation. Sustaining PA levels is necessary if the risks of long-term conditions are to be modified. However, it remains unclear whether any process benefits associated with PA, as defined above, are sustained over time or whether these are associated with moving from a sedentary lifestyle to an active lifestyle, meaning individuals accrue a 'one-off' benefit to HRQoL. One might consider that HRQoL improvements arising from improved self-esteem are transitory whereas improvements resulting from biologic mechanisms might be sustained over time. 
Notwithstanding these limitations, the findings here do indicate the potential for generating policy relevant information on promotion and evaluation of PA programmes. Programmes aimed at improving the uptake of PA might encourage participation rates by making people aware of the potential gains (which might occur sooner than later) associated with participation. In terms of economic evaluation, the findings here reinforce the need for investigation into the impact of the inclusion of such HRQoL gains. Previous analyses have suggested that including such benefits, alongside the longer-term benefits resulting from reduced incidence of long-term conditions, will lead to an improvement in the cost effectiveness of interventions designed to increase PA [35]. However, further research is required to understand the relationship between HRQoL improvements which might result directly from participation in PA as differentiated from improvements that result from sustained participation and reduced incidence of long-term conditions. Only by examining these relationships will we be in a position to accurately determine the cost effectiveness of interventions designed to promote PA.

\section{Conclusion}

Higher levels of PA are associated with better HRQoL. Using an objective measure of PA compared with subjective shows a relatively better HRQoL. Given the measurement errors associated with subjective measures, a key question is whether in order to achieve more accurate estimations of HRQoL, objective rather than subjective measures of PA ought to be favoured. We are inclined towards a cautious approach, allowing for further corroboration of the findings particularly because of discrepancy in the reference periods of data collection for the two PA measures.

\section{Competing interests}

All authors declare that they have no competing interests.

\section{Authors' contribution}

Nana Kwame Anokye and Paul Trueman undertook the analysis with support from Colin Green, drafted the first manuscript and coordinated its revision.

Toby Pavey and Rod Taylor provided an advisory role and assisted in drafting and revising the manuscript. All authors read and approved the final manuscript.

\section{HTA Funding}

This project was funded by the NIHR Health Technology Assessment programme (project number 08/72/01) See the HTA programme website for further project information. The views expressed therein are those of the authors and do not necessarily reflect those of the Department of Health.

\section{Author details}

${ }^{1}$ Health Economics Research Group (HERG), Brunel University, Uxbridge, Middlesex, UB8 3PH, England. 'Peninsula College of Medicine and Dentistry, University of Exeter, Veysey Building, Salmon Pool Lane, Exeter, EX2 4SG, England. ${ }^{3}$ School of Human Movement Studies, University of Queensland, St. Lucia Campus, Brisbane, QLD 4072, Australia.
Received: 21 February 2012 Accepted: 27 July 2012

Published: 7 August 2012

\section{References}

1. NICE: Workplace health promotion: how to encourage employees to be physically active. London: National Institute for Clinical Excellence (NICE); 2008.

2. DCMS: Media and Sport: Before, during and after: making the most of the London 2012 Games. London: Department for Culture, Media and Sport (DCMS); 2008.

3. DH: At least five a week: evidence on the impact of PA and its relationship to health. A report from the Chief Medical Officer. London: Department of Health (DH); 2004.

4. Craig R, Mindell J, Hirani V: Health Survey for England 2008. Volume 1: Physical activity and fitness. London: NHS Information Centre; 2009.

5. Chai W, Nigg CR, Pagano IS, Motl RW, Horwath C, Dishman RK: Associations of quality of life with PA, fruit and vegetable consumption, and physical inactivity in a free living, multi-ethnic population in Hawaii: a longitudinal study. International Journal of Behavioural Nutrition and Physical Activity 2010, 7(83). doi:10.1186/1479-5868-7-83.

6. Petrou S, Kupek E: Social capital and its relationship with measures of health status: Evidence from the Health Survey for England 2003. Heal Econ 2008, 17(1):127-143.

7. Bize R, Johnson JA, Plotnikoff RC: Physical activity level and health-related quality of life in the general adult population: a systematic review. Preventive Medicine 2007, 45(6):401-415.

8. Pavey T, Anokye N, Taylor A, Trueman P, Moxham T, Fox KR, et al: The effectiveness and cost-effectiveness of exercise referral schemes: $A$ systematic review and economic evaluation. Health Technology Assessment 2011, 15(44):1-254.

9. Hamer M, Stamatakis E: Objectively assessed physical activity, fitness and subjective wellbeing. Mental Health and Physical Activity 2011, 3:67-71.

10. Gillison FB, Standage M, Skevington SM: Relationships among adolescents' weight perceptions, exercise goals, exercise motivation, quality of life and leisure-time exercise behaviour: A self-determination theory approach. Health Educ Res 2006, 21(6):836-47.

11. Brand R, Schlicht W, Grossman K, Duhnsen R: Effects of a physical exercise intervention on employees'perceptions quality of life: a randomized controlled trial. Soz Praventiv Med 2006, 51(1):14-23.

12. Lindholm $\mathrm{E}$, Brevinge $\mathrm{H}$, Bergh $\mathrm{CH}$, Korner $\mathrm{U}$, Lundholm K: Relationships between self-reported health related quality of life and measures of standardized exercise capacity and metabolic efficiency in a middleaged and aged healthy population. Qual Life Res 2003, 12(5):575-582.

13. Group EQ: EuroQol-a new facility for the measurement of health-related quality of life. Health Policy 1990, 16(3):199-208.

14. Dolan P, Gudex C, Kind P: A social tariff for EuroQol: results from a UK general population survey. Discussion Paper 138. York: Centre for Health Economics; 1995.

15. O'Donovan G, Blazevich AJ, Boreham C, Cooper AR, Crank H, Ekelund U, et al: The ABC of PA for Health: a consensus statement from the British Association of Sport and Exercise Sciences. J Sports Sci 2010, 28(6):573-591.

16. Rejeski WJ, Mihalko SL: Physical activity and quality of life in older adults. J Gerontol 2001, 56(II):23-35.

17. Lubetkin El, Jia H, Franks P, Gold MR: Relationship among sociodemographic factors, clinical conditions, and health-related quality of life: Examining the EQ-5D in the US general population. Quality of Life Research 2005, 14(10):2187-96.

18. Burstrom K, Johannesson M, Diderichsen F: Swedish population health-related quality of life results using the EQ-5D. Qual Life Res 2001, 10(7):621-35.

19. Soltoft F, Hammer M, Kragh N: The association of body mass index and health-related quality of life in the general population: Data from the 2003 Health Survey of England. Qual Life Res 2009, 18(10):1293-99.

20. Sorensen J, Davidsen M, Gudex C, Pedersen KM, Bronnum-Hansen H: Danish EQ-5D norms. Scandinavian Journal of Public Health 2009, 37(5):467-74.

21. Saarni SI, Harkanen T, Sintonen H, Suvisaari J, Koskinen S, Aromaa A, et al: The impact of 29 chronic conditions on health-related quality of life: a general population survey in Finland using 15D and EQ-5D. Qual Life Res 2006, 15(8):1403-14. 
22. Sach TH, Barton GR, Doherty M, Muir KR, Jenkinson C, Avery AJ: The relationship between body mass index and health-related quality of life: Comparing the EQ-5D, EuroQol VAS and SF-6D. Int J Obes 2007, 31(1):189-96.

23. Luo N, Ko Y, Johnson JA, Coons SJ: The association of survey language (Spanish vs. English) with Health Utilities Index and EQ-5D index scores in a United States population sample. Qual Life Res 2009, 18(10):1377-85.

24. Briggs A, Clark T, Wolstenholme J, Clarke P: Missing....presumed at random: Cost-analysis of incomplete data. Health Econ 2003, 12(5):377-392.

25. Morris S, Sutton M, Gravelle H: Inequity and inequality in the use of health care in England: an empirical investigation. Social Science and Medicine 2005, 60:1251-66.

26. Austin PC, Escobar M, Kopec JA: The use of the Tobit model for analyzing measures of health status. Qual Life Res 2000, 9(8):901-10.

27. Cameron AC, Trivedi PK: Microeconometrics using Stata. Texas: Stata Press; 2009.

28. Greene W: Econometric analysis. Internationalth edition. Upper Saddle River, NJ: Pearson Prentice Hall; 2008.

29. StataCorp: Tobit analysis. 2010. http://www.ats.ucla.edu/stat/stata/dae/tobit. $\mathrm{htm}$.

30. Chatterjee S: Hadi AS, Price B: Regression analysis by example. 3rd edition. New York, US: John Wiley \& Sons Inc.; 2000.

31. Gujarati D: Basic econometrics. 3rd edition. New York: McGraw-Hill; 1995.

32. Beyler N, Nusser S, Fuller W, Gregory W: Relating self-report and accelerometer physical activity with application to NHANES 2003-2004. Proceedings of the Survey Research Methods Section:: American Statistical Association; 2010. http://www.amstat.org/sections/srms/proceedings/ allyearsf.html.

33. Penedo FJ, Dahn JR: Exercise and well-being: a review of mental and physical health benefits associated with physical activity. Curr Opin Psychiatry 2005, 18(2):189-193.

34. Saxena S, Van Ommeren M, Tang KC, Armstrong TP: Mental health benefits of physical activity. J Ment Heal 2005, 14(5):445-451.

35. Beale S, Bending M, Trueman P: An economic analysis of environmental interventions that promote physical activity. York: University of York; 2007.

doi:10.1186/1471-2458-12-624

Cite this article as: Anokye et al:: Physical activity and health related quality of life. BMC Public Health 2012 12:624.

\section{Submit your next manuscript to BioMed Central and take full advantage of:}

- Convenient online submission

- Thorough peer review

- No space constraints or color figure charges

- Immediate publication on acceptance

- Inclusion in PubMed, CAS, Scopus and Google Scholar

- Research which is freely available for redistribution 